\title{
И.В. Савин
}

Уральский федеральный университет им. первого Президента России Б.Н. Ельцина, Екатеринбург; Институт наук и технологий об окружающей среде, Автономный университет Барселоны (Universitat Autònoma de Barcelona), Испания

\section{Изучение рыночного отбора в России и мире: проблемы измерения, национальная специфика и методы стимулирования ${ }^{1}$}

Аннотация. Данная статья посвящена модели репликативной динамики как эволюционной теории конкуренции между фирмами в экономике. Мы подробно останавливаемся на способах тестирования этой модели на основе эмпирических данных, а также преимуществах и недостатках этих методов. Результаты тестирования модели репликативной динамики служат измерением интенсивности рыночного отбора в экономике. Приводится обзор исследований, тестировавших модель репликативной динамики за рубежом и в России. Делается ряд выводов о специфике функционирования рыночного отбора в России, в частности о наличии большого числа низкопроизводительных компаний (зомби-фирм). Также вносится несколько предложений о стимулировании конкуренции для ускорения экономического роста. В заключении приведены способы совершенствования модели репликативной динамики, учитывающие цепочки добавленной стоимости и сведения об изменениях оценки рыночного отбора на основе международных данных межотраслевого баланса.

Ключевые слова: конкурениия, рыночный отбор, рост фирм, репликативная динамика, зомби-фирмы.

Классификация JEL: D40, L11, L16, L50.

DOI: $10.31737 / 2221-2264-2020-48-4-9$

Мир, в котором мы живем, непрерывно развивается - иногда плавно, а иногда очень резко. Социальная сфера в целом и экономика в частности находятся в постоянном движении. Достаточно вспомнить несколько промышленных революций, которые пережило общество за последние три столетия, и как сильно за это время изменился жизненный уклад людей. Новые идеи, часто не только технологического характера, и связанные с ними инновации являются основными движущими силами этих изменений. На уровне отдельного человека или компании, а также общества в целом, такие изменения наблюдаются с разной периодичностью, и их не всегда можно предвидеть. Часто эти изменения являются результатом запланированных действий, но в некоторых случаях они возникают из-за непредвиденных побочных эффектов.

Анализ этих изменений - сложная задача, поскольку мы пытаемся понять как кра- ткосрочные, так и долгосрочные закономерности и причины возникающих изменений, природу тех участников, которые создают, внедряют, распространяют, конкурируют между собой. Чтобы решить эти вопросы, необходима концептуальная основа. Экономическая литература предлагает два альтернативных подхода для понимания описанной динамики. Первый - предлагает довольно сложную систему, построенную на понимании (порой неограниченно) рациональных агентов и определяющую равновесные состояния между ними. В рамках этого подхода экономика развивается на основе четких законов (по примеру физики) и практически всегда находится в «равновесном» состоянии, а экономические агенты действуют оптимальным для достижения наилучшего для себя результата образом, учитывая ответную реакцию своих конкурентов и делая точный прогноз будущих событий. Такой подход часто называют неоклассическим, а хоро-

\footnotetext{
1 Данное исследование поддержано грантом Президента Российской Федерации для молодых докторов наук МД-3196.2019.6.
} 
шим примером тому являются модели динамического стохастического общего равновесия. Вторая концепция представляет экономику как экосистему, которая далеко не всегда действует и реагирует предсказуемым образом; экономическая динамика является результатом деятельности, посвященной экспериментам в недостаточно понятной среде, часто сталкивающейся с неопределенностью. Этот подход в экономической литературе называют эволюционным, а одной из фундаментальных работ по этой теме является книга, написанная (Nelson, Winter, 1982).

Идея сравнения эволюционного развития экономики с эволюционным развитием живых организмов на земле далеко не нова. Считается даже, что это Чарльз Дарвин в свое время позаимствовал некоторые идеи у Адама Смита, когда формулировал теорию естественного отбора. «Теория Дарвина не защищает никаких более высоких принципов, кроме преследования индивидуумами собственных интересов, т.е. передачи собственных генов будущим поколениям. Проблема аналогична той, с которой столкнулся Адам Смит, когда он выступал за политику невмешательства государства (laissez faire) как самый верный путь к гармоничной экономике. Смит утверждал, что идеальная экономика может показаться упорядоченной и хорошо сбалансированной, но она возникнет “естественным образом" в результате взаимодействия людей, которые не следуют никаким путем, кроме преследования собственных интересов» (Gould, 1975, p. 99-100).

Проводя аналогию между природой и экономикой, случайную вариацию на генном уровне можно представить как изобретения и инновации, которые создают компании, а естественный отбор - как конкурентный рыночный отбор. В качестве единицы отбора рассматриваются не представители популяций разных видов (или внутри одного вида), а фирмы или даже целые отрасли. Ключевой моделью в рамках данного подхода стала модель репликативной динамики, впервые формализованная в (Taylor, Jonker, 1978) как модель отбора лучших стратегий поведения, в дальнейшем использовавшуюся как модель конкуренции между экономическими субъектами (Metcalfe, 1994) или, например, конкуренции между альтернативными взглядами в общественном мнении (van den Bergh, Savin, Drews, 2019). Модель репликативной динамики можно представить в качестве следующего уравнения:

$$
\Delta s_{i, t}=s_{i, t-1}\left(f_{i, t}-\bar{f}_{t}\right),
$$

где $\Delta$ - разность между двумя годами; $s_{i, t}-$ доля фирмы $i$ в период времени $t$ на рынке; $f_{i, t}$ - форма измерения приспособленности $(\text { «итнеса») })^{2}$ фирмы $i$ в период $t$ к данному рынку (это могут быть затраты на единицу продукции, качество продукта, производительность труда компании и т.д.); $\bar{f}-$ средневзвешенный (относительно долей компании на рынке) «фитнес»-фирм на рынке. Согласно уравнению (1) фирмы с «фитнесом» выше среднего должны наращивать свою долю на рынке, и наоборот. Данная модель быстро приобрела широкую популярность и используется как составная часть множества теоретических исследований (Mazzucato, 1998; Foramitti, Savin, van den Bergh, 2020).

Возникает вопрос, как оценить выполнение кажущегося столь логичным экономического закона на основе реальных данных. Существует несколько способов. Первый и наиболее распространенный из них - взять некоторый рынок³ и провести декомпозицию изменения агрегированного «фитнеса» на две компоненты:

- изменение «фитнеса» на уровне отдельно взятых фирм (within-эффект), когда фирмы наращивают качество и снижают издержки за счет иннова-

\footnotetext{
2 Здесь и далее под «фитнес» мы будем понимать меру приспособленности компаний к окружающему их миру. Слово происходит от английского слова "to fit" (подходить, приспосабливать) и получило широкое применение на основе утверждения эволюционной теории Дарвина о выживании наиболее приспособленных видов, “śurvival of the fittest”.

${ }^{3}$ За неимением четкого разделения компаний по рынкам ученые вынуждены брать классификатор экономической деятельности, как, например, отрасль номер 10 по ОКВЭД2 - «производство пищевых продуктов».
} 
ций, что приводит к росту «фитнеса» на уровне всего рынка/отрасли;

- перераспределение долей рынка среди ее участников (between-эффект), когда без какого-либо роста «фитнеса» на уровне фирм можно добиться роста агрегированного показателя.

Именно between-эффект указывает на наличие рыночной конкуренции в экономике. Для простоты интерпретации эти два эффекта нормируют в сумме к единице. Таким образом, величина каждого әффекта отражает их пропорциональный вклад в рост «фитнеса» на уровне отрасли. Этот подход применялся к разным странам, периодам времени и секторам экономики и показал очень неоднородную картину, где конкуренция могла присутствовать (в небольшой мере) в одних секторах и полностью отсутствовать в других (см. обзор литературы в (Cantner, Savin, Vannuccini, 2019)). Чаще всего оценка нормированного between-эффекта колебалась в среднем в районе нуля и за редким исключением не превышала $10 \%$ для отдельного периода или сектора экономики.

Помимо ряда замечаний, справедливых не только для данного метода, на которых мы остановимся чуть позже, очевидным недостатком метода декомпозиции является факт, что доли рынка здесь традиционно выражены не через продажи фирм, а через число занятых в них работников. Поскольку рост продаж сегодня не обязательно должен приводить к росту штата работников, данное ограничение вызывало много вопросов. Ответом на эту критику послужила прямая эконометрическая оценка уравнения (1), где в левой части уравнения стоит динамика продаж фирм, а в правой в качестве объясняющих переменных берется уровень и динамика «фитнеса» этих компаний, а также фиксированные эффекты на уровне как компаний, так и периодов времени, по которым делается оценка. Поскольку такая регрессионная модель оценивается для каждой отрасли в отдельности, влияние конкуренции на рост фирм с учетом фиксированных переменных по времени эквивалентно оценке их отклонения от среднего значения по отрасли за каждый год, что, в свою очередь, эквивалентно оценке уравнения (1). Ключевым параметром для оценки здесь является доля дисперсии роста продаж, которую удается объяснить с помощью переменных «фитнеса», т.е. насколько хорошо эти переменные позволяют объяснить динамику продаж компании. Впервые такой подход был применен в работе (Bottazzi et al., 2010) к данным по Франции и Италии, а затем (Dosi et al., 2015) - к данным по США, Германии, Франции и Великобритании. Здесь оценка вклада конкуренции в рост продаж колебалась в среднем в пределах 10-20\%, что выше оценок, полученных на основе метода декомпозиции, но все же еще очень скромная.

Третьим способом оценить роль конкуренции в экономике, который скорее дополняет, но не замещает первые два, является оценка неоднородности компаний с точки зрения их «фитнеса» в каждой отрасли и изучение того, как это значение изменяется во времени. Идея заключается в том, что при наличии здоровой экономической конкуренции разница в производительности между компаниями должна оставаться небольшой и не увеличиваться со временем. В противном случае данные свидетельствуют о том, что значительная часть компаний способна сохранять за собой рыночную нишу, даже будучи многократно менее производительными, чем их конкуренты. В литературе такие компании иногда называют «зомби-компаниями» (McGowan, Andrews, Millot, 2017) и отмечают, что их присутствие сдерживает рост экономики, поскольку эти фирмы отвлекают на себя значимую долю спроса, а также ресурсов (труда и капитала), которые могли бы быть использованы в экономике более эффективно. Например, авторы (Dosi et al., 2015) показали, что в США компания с производительностью ниже среднего по отрасли на одно стандартное отклонение в 3 раза менее производительна, чем компания, которая на одно стандартное отклонение выше этого среднего показателя. Для развивающихся экономик (таких как Индия и Китай) эта разница значительно выше и отличается в 7 раз. В Индии за последние 20 лет эта неоднородность практически не меня- 
лась, а в Китае с 1998 по 2007 г. доля низкопроизводительных фирм значимо уменьшилась (Dosi et al., 2017).

Как на этом фоне выглядит Россия? Удивительно, но до недавнего времени похожие исследования на российских данных не проводились. Впервые данные три метода анализа были проведены нами для Уральского федерального округа за период с 2006 по 2015 г. (Савин, Мариев, Пушкарев, 2019), затем для всей России - за период 2006-2017 гг. в (Савин, Мариев, Пушкарев, 2020а). За основу были взяты данные по отраслям промышленности (отрасли с 10-й по 32-ю по классификации ОКВЭД2), что скорее распространено в литературе. Авторы (Dosi et al., 2015, 2017) также проводили исследования только для отраслей промышленности. Выбор такого сегмента экономики интересен тем, что он обеспечивает диверсификацию и устойчивость экономического развития страны. Данные были взяты из базы данных «Ruslana», предоставленной Bureau van Dijk (https://www.bvdinfo.com/). Результаты декомпозиции очень напомнили результаты других стран, где вклад betweenэффекта в изменение агрегированного «фитнеса» стремится к нулю. Результаты эконометрической оценки показали более низкие значения конкурентного отбора в экономике, если учитывать только производительность труда (доля объясняющей способности - только 10-12\%). Однако если измерять «фитнес» компаний через общую факторную производительность, то оценки для России и стран ОЭСР в целом окажутся сопоставимыми. Применяя оба метода, мы также попробовали провести дифференциацию между крупными (свыше 250 сотрудников на предприятии) и малыми/средними компаниями. Оказалось, что для последних роль конкурентного отбора заметно выше. Так, например, значение between-эффекта у них в среднем равно 0,3, а для крупных компаний $-0,25$, что указывает на увеличение штата сотрудников в менее производительных крупных компаниях и противоречит логике конкурентного отбора. Эконометрический анализ показал, что если в абсолютном выражении роль конкурентного отбора на первый взгляд кажется сопоставимой для компаний разного размера в относительном выражении (т.е. насколько производительность компаний определяет их успех в сравнении с такими ненаблюдаемыми характеристиками фирм, как долгосрочные контракты с поставщиками и потребителями, монопольное положение в каком-то удаленном географическом регионе и т.д.), роль конкуренции для малых и средних предприятий в России выше. Общая доля объясненной дисперсии, в том числе с помощью фиксированных эффектов, составляет 25-27\% для малых и средних компаний и $39-41 \%$ для крупных предприятий. Это указывает на необходимость стимулирования конкуренции, особенно среди крупных компаний в России.

Однако наиболее пессимистичными получились результаты оценки дисперсии производительности компаний по отраслям и их динамика во времени. В частности, мы увидели, что разброс в производительности труда между компаниями достигает фактора 30 за рассматриваемый нами период времени (т.е. компания на одно стандартное отклонение ниже среднего в 30 раз менее производительна, чем компания на одно стандартное отклонение выше среднего). Разброс непрерывно возрастал с 2006 по 2015 г., и только в 2016-2017 гг. он несколько снизился. Стоит отметить, что на основе опроса 1000 предприятий из восьми отраслей промышленности еще в 2005-2006 гг. были выявлены более чем 20-кратные различия в производительности между компаниями одной отрасли (Голикова и др. 2007, с. 15). С тех пор ситуация ухудшилась, что свидетельствует о явном дефиците рыночных сил в российской әкономике. B (Dosi et al., 2017) мы сделали статистическую оценку того, как изменилась доля низкопроизводительных компаний в России с 2006 по 2013 г. Оказалось, что эта доля статистически значимо выросла. Данную ситуацию можно проиллюстрировать с помощью рисунка, где для отрасли ОКВЭД2 25 «Готовые металлические изделия» были построены графики плотности распределения производительности труда за 2008-2010 гг. Видно, что хвост распределения низкопроизводительных компаний 

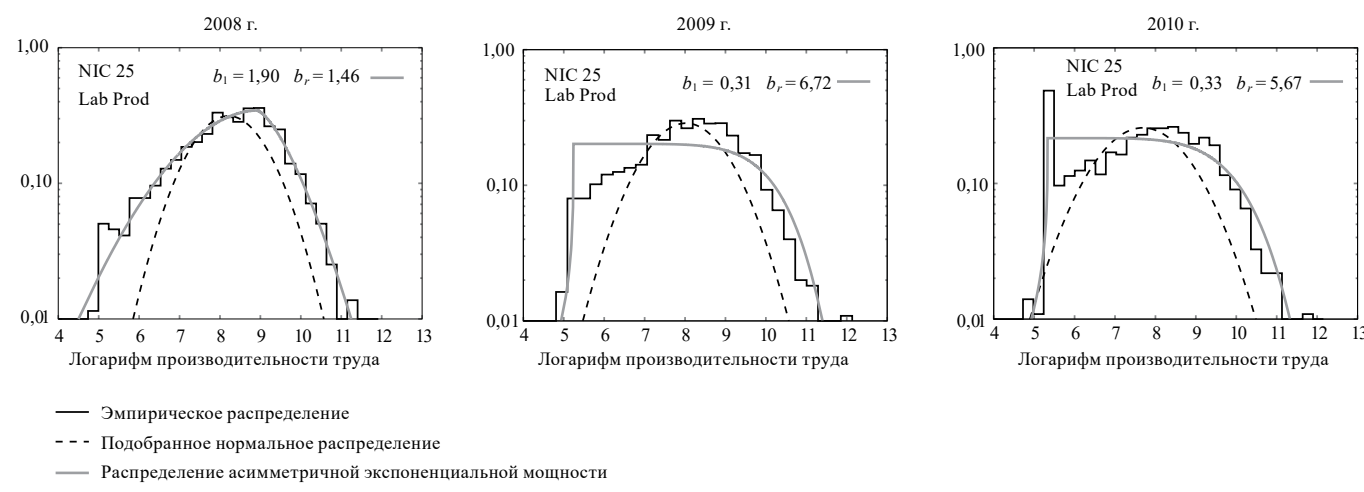

_ _ Распределение асимметричной экспоненциальной мощности

\section{Рисунок}

Графики плотности распределения производительности труда

резко увеличивается. Данная картина наблюдается практически по всем отраслям промышленности в России. Таким образом, очевидна проблема - наличие зомби-компаний в российской экономике, т.е. фирм, которые в нормальных условиях конкурентного рынка должны были бы обанкротиться, но по тем или иным причинам этого не произошло. В исследовании (Савин и др., 2020б) мы проанализировали особенности зомби-фирм в России и их роль в снижении экономического роста страны.

Рецепты борьбы с зомби-компаниями касаются преимущественно государственной экономической политики банкротства компаний и реструктурирования фирм. Эти методы обсуждались в (Andrews, McGowan, Millot, 2017), где авторы сравнивали действующие инструменты в странах ОЭСР. В частности, авторы отмечали упрощение современной процедуры банкротства (компании с высокой задолженностью и низкими показателями продаж могут объявить начало процедуры банкротства не дожидаясь, когда их положение станет еще хуже) и предлагали снижать издержки, связанные с банкротством для предпринимателей. Например, чтобы открыть после банкротства новое дело, предприниматель в Венгрии должен ждать 5 лет (тогда как в Великобритании только 1 год), что сильно снижает деловую активность в экономике, а также вероятность своевременного ухода с рынка низкопроизводительных компаний. Не стоит забывать и о работниках компаний, которые объявляют о банкротстве. Оценки вероятности для них устроиться на новую работу в течение года после увольнения разнятся в зависимости от страны и действующих в ней мер, связанных с поиском работы, переобучения и мобильности населения (так называемые «меры активного рынка труда»). Например, в Бельгии и Испании уделяется больше внимания пассивным мерам (выплате пособий по безработице), что не стимулирует занятости в экономике. Напротив, увеличение на 0,25\% ВВП расходов на меры активного рынка труда приводит к росту на 3-6\% доли работников, которые находят работу вскоре после увольнения.

При использовании и интерпретации описанных выше методов измерения конкурентного отбора важно помнить, что они имеют ряд общих недостатков, на которых хотелось бы остановиться подробнее.

Модель репликативной динамики свидетельствует о конкуренции за одного и того же потребителя, который выбирает между всеми представленными продавцами. Однако за исключением редких случаев, когда удается вручную определить всех представленных на рынке производителей (см. пример в (Cantner, Kruger, Sollner, 2012)), отсутствует реальная возможность разделить все компании по рынкам, на которых они на самом деле конкурируют друг с другом. Отраслевое разделение очень неточное. Однако даже повышение его 
детализации с двухзначного до четырехзначного (по классификатору ОКВЭД) не помогает решить проблемы (см. примеры в (Савин, Мариев, Пушкарев, 2020а)).

Вторая проблема возникает из-за неопределенности в том, что принять в качестве «фитнеса» компании. Литература знает примеры использования выручки и прибыльности на одного сотрудника, производительности труда и общей факторной производительности (ОФП), и этот список еще неполный. В (Mariev, Pushkarev, Savin, 2020) мы сравнили перечисленные выше показатели применительно к российским данным и пришли к выводу, что все они демонстрируют достаточно близкие результаты, по крайней мере для метода декомпозиции агрегированного на уровне «фитнеса» отрасли. Несколько чаще в последнее время использовался показатель производительности труда. Его преимущество состоит в том, что он, в отличие от выручки, учитывает неоднородность издержек производства, но при этом, в отличие от ОФП, не делает сильного допущения, что фирмы, применяющие разные технологии, испытывают при этом одинаковые эффекты синергии между факторами производства.

Есть еще одна причина, почему описанные выше результаты стоит воспринимать осторожно. Модель репликативной динамики предполагает, что все, что определяет успех фирмы, - соотношение ее «фитнеса» и «фитнеса» конкурентов. Но сегодня экономические агенты крайне редко самостоятельно выполняют весь цикл производства продукции или оказания услуг. Компании встраиваются в сложные цепочки создания добавленной стоимости, и, хотя в реальности потребители делают выбор между продуктами, уравнение (1) предполагает, что выбор делается между компаниями. В работе (Cantner, Savin, Vannuccini, 2019) мы предложили расширить модель репликативной динамики за счет включения в расчет «фитнеса» фирмы всех ее поставщиков. Мы проанализировали упрощенный случай, когда цепочка создания добавленной стоимости имеет одинаковое число вертикально интегрированных рынков (т.е. число звеньев цепочки добавленной стоимости строго одинаковое), и на каждом рынке в цепочке добавленной стоимости действует только одна компания, при том что «фитнес» компаний неоднороден и поменять одного поставщика на другого они не могут (например, в связи с наличием долгосрочного контракта). На этом примере мы показали, что предложенная модель способна объяснить отрицательные значения в декомпозиции для between-эффекта, даже когда существует конкуренция между компаниями на рынке конечного потребителя. Причина этого заключается в зависимости от более или менее эффективных поставщиков. Иными словами, фирма А будет проигрывать конкуренцию фирме Б, будучи сама более производительной, но имея менее конкурентных поставщиков.

Не найдя достаточно детализированных данных на уровне фирм для эмпирической проверки, предложенной в (Cantner, Savin, Vannuccini, 2019), расширенной модели репликативной динамики, мы провели исследование на основе международных данных межотраслевого баланса (World Input-Output Data). Согласно этим данным в каждом конкретном секторе экономики страны конкурируют за глобальную долю рынка, и мы можем рассчитать добавленную стоимость и издержки труда, связанные с ее созданием, которые из отдельной отрасли одной страны поступают в форме товаров и услуг в определенную отрасль другой страны (Cantner et al., 2020). Полученные оценки показывают существенное увеличение как значения between-эффекта, так и доли дисперсии роста продаж, объясняемой факторами производительности труда. Это указывает на то, что представленные ранее оценки конкурентного отбора могут быть заниженными в абсолютном выражении. Иными словами, оценка between-эффекта, полученная для России ранее и колеблющаяся в районе нуля, а также объясняющая способность факторов «фитнеса» в регрессионной модели на уровне 10-12\%, скорее всего ниже, чем если бы мы провели оценку модели репликативной динамики для России с учетом цепочек добавленной стоимости. Однако это не отменяет того 
факта, что для России эти оценки все же ниже, чем для ряда стран ОЭСР, и что проблема низкопроизводительных зомби-фирм в нашей стране выражена во всех отраслях промышленного производства, а значит, экономика использует свои ресурсы менее эффективно, чем если бы зомби-компании ушли с рынка.

\section{ЛИТЕРАТУРА / REFERENCES}

Голикова В.В., Яковлев А.А., Кузнецов Б.В., Ясин Е.Г., Гончар К. (2007). Российская промышленность на перепутье. Что мешает нашим фирмам стать конкурентоспособными. Научный доклад ГУ ВШЭ. Режим доступа: https:/ /id.hse. ru/books / 47767969.html [Golikova V.V., Jakovlev A.A., Kuznetsov B.V., Jasin E.G., Gonchar K. (2007). Russian industry at the Crossroads: What prevents our firms from being competitive. The Report by SUHSE. Available at: https://id.hse.ru/ books /47767969.html (in Russian).]

Савин И.В., Мариев О.С., Пушкарев А.А. (2019). Выживает сильнейший? Измерение конкурентного отбора на примере Уральского федерального округа // Экономический журнал ВШЭ. Т. 23. № 1. C. 90-117. Режим доступа: https:// ej.hse.ru/en/2019-23-1/252584141.html [Savin I.V., Mariev O.S., Pushkarev A.A. (2019). Survival of the fittest? Measuring the strength of market selection on the example of the Urals federal district. The HSE Economic Journal, 23, 1, 90-117. Available at: https://ej.hse.ru/en/2019-231/252584141.html (in Russian).]

Савин И.В., Мариев О.С., Пушкарев А.А. (2020а). Оценка рыночного отбора в России: когда размер (фирмы) имеет значение // Вопросы экономики. № 2. C. 101-124. DOI: 10.32609/0042-87362020-2-101-124 [Savin I.V., Mariev O.S., Pushkarev A.A. (2020a). Measuring the strength of market selection in Russia: When the (firm) size matters. Voprosy Ekonomiki, 2, 101-124. DOI: 10.32609/00428736-2020-2-101-124 (in Russian).]
Савин И.В., Мариев О.С., Пушкарев А.А. (2020б). «Зомби-компании» в России: их специфика и роль в экономике. Неопубликованный препринт. [Savin I.V., Mariev O.S., Pushkarev A.A. (2020b). "Zombie companies" in Russia: Their specificity and role in the economy. Unpublished paper (in Russian).]

Andrews D., McGowan M.A., Millot V. (2017). Confronting the zombies: Policies for productivity revival. OECD economic policy papers 21. OECD Publishing. DOI: 10.1787/ f14fd801-en

Bottazzi G., Dosi G., Jacoby N., Secchi A., Tamagni F. (2010). Corporate performances and market selection: Some comparative evidence. Industrial and Corporate Change, 19, 1953-1996.

Cantner U., Inoue H., Mundt P., Savin I., Vannuccini S. (2020). Market selection in global value chains. Unpublished working paper.

Cantner U., Kruger J., Sollner R. (2012). Product quality, product price, and share dynamics in the German compact car market. Industrial and Corporate Change, 21 (5), 1085-1115.

Cantner U., Savin I., Vannuccini S. (2019). Replicator dynamics in value chains: Explaining some puzzles of market selection. Industrial and Corporate Change, 28 (3), 589-611. DOI: $10.1093 /$ icc/dty060

Dosi G., Luna I., Mathew N., Netto E.Y.H., Savin I., Yu X. (2017). Productivity, market selection and corporate growth: Comparative evidence from BRIC nations. Proceedings of the 5th CONCORDi conference in Seville, September 2017.

Dosi G., Moschella D., Pugliese E., Tamagni F. (2015). Productivity, market selection, and corporate growth: Comparative evidence across US and Europe. Small Business Economics, 45, 643-672.

Foramitti J., Savin I., van den Bergh J. (2020). Emission tax vs. permit trading under bounded rationality and dynamic markets. Energy Policy. Forthcoming. DOI: 10.1016/j.enpol.2020.112009

Gould S.J. (1975). Ever since Darwin: Reflec- 
tions in natural history. N.Y.: Norton \& Company.

Mariev O.S., Pushkarev A.A., Savin I.V. (2020). Estimating market selection in Russia: Comparative analysis of different performance indicators. In: 8th International Conference on Innovation Management, Entrepreneurship and Sustainability IMES 2020. May 28-29, 2020 at the University of Economics in Prague.

Mazzucato M. (1998). A computational model of economies of scale and market share instability. Structural Change and Economic Dynamics, 9 (1), 55-83.

McGowan M.A., Andrews D., Millot V. (2017). The walking dead? Zombie firms and productivity performance in OECD countries OECD Economics Department. Working Papers No. 1372.
Metcalfe J.S. (1994). Competition, Fisher's principle and increasing returns in the selection process. Journal of Evolutionary Economics, 4 (4), 327-346.

Nelson R.R., Winter S. (1982). An evolutionary theory of economic change. Cambridge: Harvard University Press.

Taylor P., Jonker L. (1978). Evolutionary stable strategies and game dynamics. Mathematical Biosciences, 40, 145-156.

Van den Bergh J.C.J.M., Savin I., Drews S. (2019). Evolution of opinions in the growth-vs-environment debate: Extended replicator dynamics. Futures, 109, 84-100. DOI: 10.1016/j.futures.2019.02.024

Поступила в редакиию 00.00.2020

Received 00.00.2020

\section{I.V. Savin}

Ural Federal University named after the first President of Russia B.N. Yeltsin, Yekaterinburg, Russia; Institute of Environmental Science and Technology (ICTA), Autonomous University of Barcelona, Spain

\section{Studying market selection in Russia and abroad: Measurement problems, national specificity and stimulating methods ${ }^{4}$}

Abstract. This article is devoted to the model of replicator dynamics as an evolutionary theory of competition between firms in economics. We describe in detail how to test this model based on empirical data, as well as the advantages and disadvantages of these methods. The results of testing the replicator dynamics model serve as a measure of the intensity of market selection in an economy. An overview of studies that have tested the replicator dynamics model abroad and in Russia is given. A number of conclusions is drawn about the specificity of functioning of market selection in Russia, in particular, about the presence of a large number of low-performing companies (zombie firms). Several proposals are also being made to stimulate competition in order to accelerate economic growth. In the conclusion, we suggest ways on how to improve the model of replicator dynamics taking into account value chains, and provide information about changes in the assessment of market selection based on world input-output data.

Keywords: competition, market selection, firm growth, replicator dynamics, zombie firms.

JEL Classification: D40, L11, L16, L50.

DOI: $10.31737 / 2221-2264-2020-48-4-9$

\footnotetext{
4 This research has been supported by the grant from the President of the Russian Federation for young doctors of science MD-3196.2019.6.
} 